\title{
Hong Kong: un tigre sin garras
}

\section{I}

\section{ntroducción}

A cinco años del traspaso de Hong Kong, por parte de Gran Bretaña, a China, ocurrido el primero de julio de 1997, parece ser que las cosas no han funcionado del todo bien ni para el gobierno de la Región Administrativa Especial de Hong Kong ni para el gobierno de la República Popular de China. Después de casi un siglo bajo el dominio de los ingleses, Hong Kong llegó a convertirse en uno de los lugares más prósperos de Asia y una de las regiones económicamente más dinámicas del planeta, caracterizada por la convivencia chinos y extranjeros, es decir, la convergencia de las culturas oriental y occidental, con una gran diversidad en su composición étnica.

Gracias a su privilegiada ubicación geográfica, las óptimas condiciones naturales para un puerto natural, el favorable ambiente internacional, la influencia de los ingleses por un prolongado periodo de tiempo y una eficiente estrategia de desarrollo, la isla se convirtió rápidamente en el centro internacional de finanzas, de comercio, de transporte marítimo, de información y turismo del lejano oriente. Para mediados de los años noventa, se le llegó a considerar como el primer puerto de contenedores del mundo, el cuarto centro bancario y mercado de oro, el quinto mercado de divisas, el octavo mercado de valores y ente comercial y económico. ${ }^{1}$

Para 1994, Hong Kong alcanzó un producto interno bruto (PIB) per cápita de 23 mil dólares, lo que lo ubicaba en el sexto lugar del mundo, por arriba de países desarrollados como la misma Gran Bretaña, Canadá, Italia y Australia. ${ }^{2}$ Sin embargo, desde finales de los años noventa se presentaron ciertos problemas de carácter económico y político, como un reflejo

* Investigador del Departamento de Estudios del Pacífico de la Universidad de Guadalajara. de que las cosas parecían no estar funcionando como antes.

\section{La llegada de los ingleses}

Antes de la invasión y ocupación de esta isla por los ingleses, Hong Kong había alcanzado un incipiente desarrollo socioeconómico, cultural y educativo como parte del territorio chino. De hecho, el control de la región de Hong Kong por las autoridades chinas se remonta a la dinastía Qing (221-206 antes de nuestra era), cuando los gobiernos centrales de las dinastías Yuan, Ming y Qing establecieron allí departamentos de supervisión militar, ejerciendo desde entonces la administración de la isla mediante ciertos organismos de gobierno.

El problema sobre Hong Kong comenzó en el siglo XVIII cuando los ingleses entraron en conflicto con los chinos a causa del contrabando de opio, el cual se cultivaba en la India y se distribuía en todo el territorio de China a cambio de seda, especias, plata, jade y otras joyas, convirtiéndose pronto en un lucrativo negocio para los ingleses y un serio problema social para los chinos. El 10 de marzo de 1839, por órdenes del emperador Dao Guang se prohibió el comercio de opio y se exigió a los extranjeros -la mayoría de ellos ingleses- que entregaran todo el opio que poseían a las autoridades chinas, quienes arrojaron cerca de 20,000 cajas de ese producto al mar. ${ }^{3}$

En Inglaterra, este hecho fue considerado como una ofensa y una abierta provocación de los chinos para frenar el comercio y, sobre todo, la presencia de los ingleses en la región, por lo que en respuesta fue enviada la armada británica hacia Hong Kong, destrozando sin mayores problemas a una débil armada china en noviembre del mismo año; posteriormente atacaron Cantón, ocuparon Shanghai y llegarían hasta Nanking. Estos sucesos serían conocidos como la Guerra del Opio. ${ }^{4}$ 
Como resultado de los anteriores acontecimientos, en 1842, el gobierno de Qing se vio obligado a firmar el Tratado de Nanking, mediante el cual los chinos tuvieron que ceder a manera de compensación la isla de Hong Kong a Inglaterra. Este sería el primer tratado firmado en la historia moderna de China, con lo que se vería reducida al status de sociedad semicolonial y semifeudal. Posteriormente, entre 1856 y 1860, Inglaterra y Francia entraron en guerra con China nuevamente por el comercio del opio, tras lo cual los ingleses ocuparon la península de Kowloon y obligaron al gobierno de Qing a firmar un segundo tratado, conocido como el Convenio de Pekín, que delimitaba por la fuerza la parte sur de la península como Colonia de Hong Kong. ${ }^{5}$

En 1898, bajo el pretexto de que la ocupación de la bahía de Cantón (Guangzhou) por parte de Francia constituía una amenaza para Hong Kong, los ingleses obligaron a Qing a firmar un tercer tratado conocido como Convenio para la Extensión del Territorio de Hong Kong, mediante el cual se concesionó a los ingleses por un plazo de 99 años un vasto sector del territorio chino que incluía 235 islas adyacentes a Hong Kong, que se llamaron los Nuevos Territorios. ${ }^{6}$

A partir de ese momento Inglaterra gobernó a Hong Kong en una forma típicamente colonial. Sin embargo, el gobierno chino siempre se negó a reconocer a la isla como una colonia inglesa, puesto que para él este término sólo podía aplicarse a un país que perdía su soberanía al quedar dominado y gobernado por otro país, por lo que en el caso de Hong Kong, al formar parte del territorio chino, no podía considerársele como colonia.

\section{El traspaso de Hong Kong a China}

No obstante los anteriores tratados a que prácticamente fue sometido el gobierno de Qing, los sucesivos gobiernos de China se opusieron firmemente a la ocupación permanente de Hong Kong por parte de Inglaterra, razón por la cual enfocaron sus esfuerzos a recuperar ese territorio y los adyacentes. Con la proclamación de la República Popular de China en 1949, el gobierno chino reforzó su posición en el sentido de considerar a Hong Kong como su territorio y de recuperar su control en un futuro próximo, por lo que a partir de ese momento dejaron de reconocer los tres tratados impuestos por los ingleses y abogaron por resolver el problema por la vía de las negociaciones en los más altos foros internacionales. Fue así como, en 1972, a petición del gobierno chino, la Organización de las Naciones Unidas (ONU) acordó excluir a Hong Kong y Macao de su lista de colonias. ${ }^{7}$

En 1978, China inició un proceso de profundas reformas políticas y económicas que contemplaban tres tareas fundamentales: la modernización socialista, la reunificación pacífica del país y la lucha contra el hegemonismo y el imperialismo. Ala luz de estas nuevas ideas, el dirigente chino Deng Xiaoping, formuló el concepto de "un país, dos sistemas" como una vía para resolver la situación de Taiwan y Hong Kong. Al mismo tiempo, al aproximarse la fecha de expiración del periodo de arrendamiento de los Nuevos Territorios en 1997, Inglaterra había empezado a sondear la posición y actitud del gobierno comunista chino en torno a la resolución del problema de Hong Kong.

Bajo estas circunstancias, entre 1982 y 1984, los gobiernos chino e inglés sostuvieron negociaciones sobre el futuro de Hong Kong, 
realizándose éstas en dos etapas. En la primera, de septiembre de 1982 a junio de 1983, cuando la primera ministra Margaret Thatcher visitó China, las dos partes conversaron esencialmente sobre los principios y procedimientos. En la segunda etapa, de julio de 1983 a septiembre de 1984, las delegaciones de los respectivos gobiernos efectuaron 22 rondas de conversaciones sobre cuestiones específicas en torno al traspaso. 8

Durante el periodo inicial de las negociaciones, Inglaterra planteó su deseo de preservar la validez de los tratados. Sin embargo, en septiembre de 1982, al reunirse con Margaret Thatcher, Deng Xiaoping manifestó de manera tajante la postura del gobierno chino para la solución del problema de Hong Kong, a partir de tres principios básicos: en primer lugar, que el problema de Hong Kong podía ser objeto de negociaciones entre ambos países, sin embargo, al considerar a la isla como territorio chino no aceptaba discutir la cuestión de su soberanía, por lo que China tendría que recuperar a Hong Kong en 1997. En segundo lugar, si durante el periodo de transición (desde el inicio de las negociaciones hasta 1997) surgiera una situación fuera de control, las autoridades chinas se verían obligadas a considerar el momento y la forma de recuperar la isla. En tercer lugar, después de la recuperación, China aplicaría una política apropiada en Hong Kong a fin de mantener su prosperidad y estabilidad, la cual incluía la creación de la Región Administrativa Especial de Hong Kong, que preveía su administración por parte de los chinos locales y el mantenimiento sin cambio del sistema socioeconómico.

Ante la firmeza de la postura china, la primera ministra británica manifestó que las dos partes podrían buscar un acuerdo permanente en torno a Hong Kong con base en la propuesta de China, por lo que Inglaterra no insistiría más en la administración de la isla, ni buscaría ninguna forma de administración conjunta con China sobre la misma. Con ello, se allanaba el principal obstáculo de las negociaciones chinobritánicas para la restitución tanto de la soberanía como de la administración de Hong Kong hacia China a partir del primero de julio de 1997.

Fue así como China e Inglaterra acordaron establecer el Grupo Conjunto Chino-Británico de Enlace. Este grupo se encargó de discutir los asuntos relativos a la transferencia del poder político en 1997, así como de intercambiar información y llevar a cabo consultas acerca de los problemas acordados por las dos partes. Básicamente, las rondas finales de las negociaciones se centraron sobre temas como la nacionalidad, la aviación civil, la tierra y otros problemas específicos de carácter político y técnico, efectuándose repetidas consultas para la redacción del texto de un acuerdo.

El 18 de septiembre de 1984, finalmente China e Inglaterra llegaron a un acuerdo en todos los problemas tratados y el 19 de diciembre del mismo año se firmó oficialmente en Pekín, lo que sería la Declaración Conjunta del Gobierno de la República Popular de China y el Gobierno del Reino Unido de Gran Bretaña e Irlanda del Norte sobre el problema de Hong Kong, dando por concluidas las negociaciones iniciadas apenas dos años atrás.

En la Declaración Conjunta se estableció que el gobierno de la República Popular de China reanudaría el ejercicio de su soberanía sobre Hong Kong a partir del primero de julio de 1997, además de exponer sus principios y políticas fundamentales, los cuales consistían básicamente en los siguientes puntos:

1. Instaurar la Región Administrativa Especial de Hong Kong subordinada directamente al Gobierno Popular Central.

2. La Región Administrativa Especial de Hong Kong disfrutaría de un alto grado de autonomía, excepto en los asuntos relacionados con la defensa y las relaciones exteriores, los cuales incumben al Gobierno Popular Central.

3. Permanecerían inalteradas en lo fundamental las leyes vigentes en Hong Kong.

4. El gobierno de la Región Administrativa Especial de Hong Kong estaría integrado por residentes locales. 
5. El sistema social y económico y el modo de vida existentes en Hong Kong se conservarían sin cambio.

6. La Región Administrativa Especial de Hong Kong mantendría el status de puerto libre y de territorio separado en el aspecto arancelario y el status de centro financiero internacional, así como sus finanzas independientes.

7. La Región Administrativa Especial de Hong Kong podría, bajo el nombre de "Hong Kong, China", mantener y desarrollar por cuenta propia relaciones económicas y culturales con diversos países y regiones y con las organizaciones internacionales pertinentes.

8. El orden público de Hong Kong estaría a cargo del gobierno de la Región Administrativa Especial.

9. Finalmente, se expone que estos principios y políticas fundamentales permanecerían inalterados por un periodo de 50 años. ${ }^{9}$

\section{Implicaciones políticas del traspaso}

Una vez que el gobierno chino reasumió la soberanía sobre Hong Kong, de acuerdo con lo establecido en la Ley Fundamental formulada expresamente para este territorio, se convirtió en una región administrativa con un alto grado de autonomía política, pero subordinada directamente al gobierno de la República Popular de China. La Ley Fundamental de Hong Kong estipula, entre otras cosas, que la isla contará con sus propios poderes ejecutivo, legislativo y judicial independientes, por lo que, tanto los departamentos del Gobierno Popular Central, así como las provincias, regiones autónomas y municipios directamente subordinados a este mismo, no pueden interferir en los asuntos políticos y económicos que la Región Administrativa Especial de Hong Kong maneje por su cuenta.

La Ley Fundamental también establece que las leyes formuladas por la legislatura de la región deben ser turnadas al Comité Permanente de la Asamblea Popular Nacional China únicamente para fines de registro, lo que no pone en riesgo su entrada en vigor. En todo caso, si este órgano de gobierno, tras haber consultado con la Comisión para la Ley Fundamental de la Región Administrativa Especial de Hong Kong bajo su subordinación, considera que alguna ley elaborada por la legislatura de la región contraviene a lo estipulado en la Ley Fundamental, respecto a los asuntos a cargo de las autoridades centrales o a las relaciones entre éstas y las autoridades de la región, podrá regresar la ley en cuestión para su revisión, más no enmendarla. De igual forma, las leyes nacionales no se pueden aplicar en la Región Administrativa Especial de Hong Kong, excepto aquellas relacionadas con la defensa y los asuntos exteriores de la República Popular de China. ${ }^{10}$

\section{Los últimos cinco años}

A cinco años de haberse reintegrado a China, Hong Kong atraviesa por momentos difíciles como consecuencia tanto de la crisis económica que se propagó a varias economías de la región a fines de los años noventa y del posterior ambiente recesivo de la economía mundial, como por la pérdida de confianza, particularmente de los sectores más conservadores, hacia el Gobierno Popular Central, por su aparente incumplimiento del lema que consagró el retorno de la ex colonia británica a la soberanía china: "un país, dos sistemas". Con este enfoque se ha pretendido mantener intactas las formas de organización política y económica de un territorio considerado como adalid del capitalismo y del libre mercado, y que goza de mayores libertades civiles y políticas que el resto de la República Popular de China.

En el terreno económico, una vez superados los efectos de la crisis financiera asiática, el PIB de Hong Kong registró un crecimiento de 10 por ciento en el año 2000. Sin embargo, debido a los recientes acontecimientos -el ambiente de recesión de la economía de Estados Unidos, el clima de inseguridad provocado por el terrorismo y la incertidumbre de los mercados financieros- que han afectado a la economía internacional, se prevé una drástica caída en el crecimiento económico de la isla durante los próximos años. Quizás el indicador que mejor refleja el deterioro de la economía 
hongkonesa es el PIB per cápita, el cual mostró un descenso de 26,103 dólares por habitante, previos a 1997 , a 25,590 dólares para el $2000 .^{11}$

Por otra parte, en un territorio donde se rehúsa una y otra vez legislar un salario mínimo, la distancia entre ricos y pobres se ha ido ensanchando cada vez más. Cabe mencionar que en Hong Kong coexisten alrededor de mil familias de multimillonarios y un amplio sector de trabajadores no calificados y otro tanto de desempleados, que alcanzan una tasa de 7.4 por ciento. ${ }^{12}$ También existe una fuerte preocupación de que, a medida que Hong Kong reestructura su economía-el cambio de un modelo basado en las manufacturas tradicionales hacia otro basado en las nuevas tecnologías de la información y de las telecomunicaciones-, el desempleo y las diferencias de riqueza se verán incrementadas.

En este sentido, el gobierno de Hong Kong admite que, desde el traspaso de su soberanía, ha tenido que adoptar una política de mayor intervención en la economía del territorio para hacer frente a la fuerte recesión que trajo consigo la crisis financiera asiática, lo que los ha obligado a abrirse a nuevas ideas e incluso a diseñar una política industrial activa para reorientar la economía de la isla. Hecho que representa un cambio radical respecto a la política de no intervención del pasado. ${ }^{13}$

Otro suceso que ha puesto de manifiesto el paulatino debilitamiento de la economía de Hong Kong, es su aparente pérdida de importancia como centro de negocios a nivel mundial. Recientemente, en su clasificación anual, la Economist Intelligence Unit (EIU) de Londres degradó a Hong Kong del primero al sexto lugar como centro mundial de negocios, bajo el argumento de que las oportunidades de negocios de la isla habían menguado desde que China recuperó la soberanía de la ex colonia británica. Según la EIU, en los últimos años se han presentado ciertas controversias que reflejan la erosión gradual del marco de legalidad en Hong Kong y de su reputada política económica delaissez-faire. ${ }^{14}$
Incluso, voces aún más pesimistas predicen que Hong Kong, la otrora considerada puerta de entrada a China, parece ser que ha llegado al final de su época dorada, ya que el gigante asiático desde que inició sus reformas económicas ha estado abriendo muchas otras puertas al exterior a lo largo de su territorio, entre las que destacan Shanghai y Shenzhen. ${ }^{15}$

Mientras tanto, en el terreno político, las primeras dudas sobre la autonomía legislativa de Hong Kong aparecieron en 1999, cuando el gobierno local solicitó al Gobierno Popular Central reinterpretar la Ley Fundamental, a fin de modificar una decisión del Tribunal Judicial de Hong Kong sobre el derecho de residencia. Ello debido a que dicha instancia judicial había aprobado que alrededor de 1.6 millones de chinos hijos de residentes de la isla tenían derecho a instalarse con sus progenitores en la misma. ${ }^{16}$

Empero, a petición del gobierno hongkonés, temeroso de ver una avalancha de nuevos ciudadanos en un territorio ya de por sí superpoblado -siete millones de personas viviendo en una superficie de $1,100 \mathrm{~km}^{2}$, y con densidades de población de hasta un cuarto de millón de habitantes por kilómetro cuadrado-, el Parlamento chino examinó la resolución judicial y revocó la decisión. Este hecho fue interpretado por muchos habitantes de la isla como una flagrante injerencia de Pekín en los asuntos de Hong Kong, que pone en entredicho la supuesta autonomía política de esta última, $y$ que ha generado diversos incidentes relacionados con los inmigrantes de origen chino. ${ }^{17}$

Otros casos que han llamado mucho la atención y que han generado fuertes controversias respecto al desempeño del gobierno local fueron la concesión, en marzo de 1999 , de un proyecto inmobiliario vinculado a las industrias de las nuevas tecnologías -valorado en 1.6 mil millones de dólares- a una empresa privada controlada por un magnate de la construcción hongkonés, sin concurso público de por medio como tradicionalmente se venía realizando. Posteriormente, a principios del 2000, las empresas Cyberworks - de capital 
hongkonés- y Singapore Telecom - propiedad del gobierno de Singapur-, contendieron por la compra de Cable \& Wireless HKT, la principal empresa de telecomunicaciones de Hong Kong. ${ }^{18}$ $\mathrm{Al}$ final fue Cyberworks quien resultó ganadora; sin embargo, el proceso de adquisición se vio empañado por acusaciones, en el sentido de que algunos funcionarios de los gobiernos de China y de Hong Kong se habían coludido con altos directivos de la empresa Hong Kong Telecom para mantener la firma en manos de capital local. Aun cuando el gobierno local sostiene que las acusaciones de intervención política son infundadas, se percibe una creciente desconfianza entre los inversionistas locales y extranjeros, así como de los ciudadanos en general, quienes consideran que con tales acciones se está poniendo en riesgo todo un modelo de desarrollo.

De acuerdo con una encuesta realizada por la Universidad de Hong Kong, tres de cuatro habitantes consideran que las condiciones generales de Hong Kong han empeorado desde su reunificación con China y que el grado de optimismo de la población respecto al futuro de la Región Administrativa Especial ha descendido de 60 a 26 por ciento, como un reflejo de la menor confianza de la población en general hacia las autoridades tanto locales como de China. ${ }^{19}$

\section{Conclusiones}

El famoso lema "un país, dos sistemas", formulado en 1978 por el promotor de las reformas económicas Deng Xiaoping, con miras a la reunificación de Hong Kong (y probablemente también de Taiwan) con China, como una suerte de compromiso para respetar las formas de organización política y económica prevalecientes en la isla una vez reintegrada a la soberanía china, ha levantado muchas dudas. A cinco años del traspaso de la ex colonia británica a la soberanía china, se perciben algunas señales de que Pekín está buscando de manera paulatina una mayor presencia en los asuntos económicos y políticos de la isla, algo que los hongkoneses no están dispuestos a aceptar. Al mismo tiempo, se aprecian signos de deterioro en la economía de Hong Kong como consecuencia, por un lado, de la misma apertura económica de China y por el otro, de la recesión en la economía mundial, aunados al clima de incertidumbre que perciben los habitantes respecto al futuro de la isla.

\section{Notas}

1 De acuerdo con información de la Embajada de la República Popular de China, contenida en el documento "Hong Kong: perla brillante de oriente", http:// www.copesa.cl/Casos/HK97

2 The World Bank, "1996 World Bank Atlas", Washington, D.C.

3 "Hong Kong, del opio al dólar", en La Revista, http:// www.elmundo.es/larevista/num85.

4 Ibid.

5 Embajada de la República Popular de China, "El origen del problema de Hong Kong”

6 Ibid.

7 Embajada de la República Popular de China, "Hong Kong no es colonia británica", http://www.copesa.cl

8 Véase "El fin de la última colonia británica", http:// www.copesa.cl/Casos/HK97

9 Ibid.

10 Embajada de la República Popular de China, "Traspaso de Hong Kong a China”, http://www.copesa.cl/Casos/ HK97

11 The World Bank, "2002 World Development Indicators”, Washington, D.C.

12 Véase "Depression settles over Hong Kong", en The Guardian Unlimited, June 29, 2002, http:// www.guardian.co.uk

13 Véase "Hong Kong abraza la nueva economía", en Asiared. Economía, política y cultura de Asia, junio de 2000, http://www.asiared.com/historia. "Hong Kong goes proactive", en Far Eastern Economic Review, March 28, 2002.

14 Ibid.

15 Véase "Shanghai y Hong Kong: rivales y complementarias", en Asiared. Economía, política y cultura de Asia, mayo de 2001, http://www.asiared.com/ historia

16 Véase "Los empresarios chinos se forman a la occidental", en Asiared. Economía, política y cultura de Asia, julio de 1999, http://www.asiared.com/historia

17 "Migrants defy order to leave Hong Kong", en The Guardian Unlimited, April 3, 2002, http:// www.guardian.co.uk/china/story

18 Asiared, op. cit., junio de 2000.

19 The Guardian Unlimited, op. cit., June 29, 2002; Far Eastern Economic Review, "The will of the people unheard”, April 11, 2002. 